\title{
Diseño de un prototipo de calentador solar con materiales reciclados
}

\section{Design of a prototype of a solar heater with recycled materials}

\author{
Víctor Ávila ${ }^{1}$, Rosa Chong ${ }^{1 *}$, Suyen Terreo ${ }^{l}$, Nacarí Marín ${ }^{2}$ \\ ${ }^{1}$ Licenciatura en Ingeniería Electromecánica - Centro Regional de Azuero - Universidad Tecnológica de Panamá \\ ${ }^{2}$ Universidad Tecnológica de Panamá, Centro Regional de Azuero, Facultad de Ingeniería Mecánica
}

Resumen El presente artículo describe el diseño de un prototipo de calentador de agua hecho con materiales reciclados y que aprovecha la radiación térmica. Se presentan los métodos y materiales utilizados para el desarrollo del prototipo del calentador solar, así como su funcionamiento teórico. En el diseño se considera la orientación que este debe llevar para su máximo aprovechamiento. En las pruebas realizadas, se confirma que los cuerpos negros absorben la radiación solar, y el efecto invernadero que provoca el vidrio.

Palabras clave Calentador de agua, efecto invernadero, materiales reciclados, radiación térmica.

\begin{abstract}
This article describes the design of a prototype for a water heater made with recycled materials and takes advantage of thermal radiation. The methods and materials used for the development of the solar heater prototype are presented, as well as its theoretical operation. The design considers the best orientation for maximum use. In the tests carried out, it is confirmed that black bodies absorb solar radiation, as well as the greenhouse effect caused by glass.
\end{abstract}

Keywords Water heater, greenhouse effect, recycled materials, thermal radiation.

* Corresponding author: rosa.chong@utp.ac.pa

\section{Introducción}

Cada día más se necesitan formas de energía que sean amigables con el ambiente y que sean rentables. Una manera de lograr este objetivo es reutilizar y aprovechar materiales de desecho; muchos de ellos se pueden aprovechar a partir de las energías renovables.

A través de este artículo se presentan los componentes del calentador de agua solar, así como los materiales y métodos utilizados para llevar a cabo el desarrollo de un prototipo de calentador de agua. También se incluye el funcionamiento del calentador y cómo se debe colocar para su mayor aprovechamiento.

El objetivo de esta investigación es proponer un diseño de calentador solar con materiales reciclados y hacer un estudio para comprobar si los materiales utilizados favorecen la transferencia de calor al agua y qué tanto tiempo se mantiene el agua caliente.

\section{Calentador solar}

Un captador solar plano es una tecnología solar térmica de baja temperatura $\left(\mathrm{T}<90^{\circ} \mathrm{C}\right)$ que se destina para agua caliente destinada al consumo humano.
Los subsistemas empleados en esta tecnología dependen del tipo de instalación, pero en general, pueden señalarse los siguientes:

- El subsistema de captación de esta tecnología está constituido por los colectores térmicos planos o paneles solares térmicos planos, pertenecientes al grupo denominado sin concentración, es decir, a aquellos que utilizan la energía solar con la misma intensidad con la que incide. Los colectores planos captan la energía solar, transformándola en energía térmica e impidiendo su salida al exterior (efecto invernadero).

- El subsistema de acumulación tiene la finalidad de almacenar el agua caliente para suministrarla en el momento que se requiera.

- El subsistema intercambiador independiza el agua que circula por el captador del agua de consuma.

- El subsistema auxiliar está compuesto por un conjunto de elementos y dispositivos, tales como tuberías de conducción, válvulas, bombas, purgadores, energía auxiliar, etc.

Las instalaciones se pueden clasificar también por su principio de circulación en: 
- Instalación solar térmica de circulación forzada, estas van equipadas con dispositivos (bombas) que provocan la circulación forzada del agua.

- Instalación solar térmica de circulación natural (termosifón), el fluido circula por convección natural. Tiene menos elementos y es más barata [1].

El diseño propuesto consiste en una instalación solar térmica de circulación natural.

La orientación del calentador depende del hemisferio en donde se encuentre, mientras que la inclinación del dispositivo depende de la latitud [2].

\section{Materiales y Métodos}

Los materiales y métodos a utilizar para el desarrollo del prototipo del calentador, se detallan a continuación.

\subsection{Materiales e instrumentos de medición}

Termómetro digital: son instrumentos que se utilizan para medir temperatura y que tienen una pantalla digital y una sonda permanente. Utiliza un sensor basado en termistores, dispositivos electrónicos de estado sólido cuyas características eléctricas cambian con la temperatura. Son portátiles. Pueden ofrecer la temperatura en grados Fahrenheit, centígrados o Celsius. También pueden mostrar la amplitud y la escala.

Termómetro analógico: es un tubo de vidrio en cuyo interior hay mercurio, mismo que se desplaza uniformemente dependiendo de la temperatura [3-4].

Mangueras (3 tipos):

1. Manguera de riego. Su rango de operación: presión de compensación (7 a 60 psi), temperatura del agua hasta $43.3^{\circ} \mathrm{C}[5]$.

2. Manguera para nivel. Material de la manguera es PVC, diámetro interior de manguera $3 / 8$ pulg., color de manguera trasparente, presión máxima 71 psi a 25 grados Celsius, característica ideal para temperaturas de 10 a $60^{\circ} \mathrm{C}$. Conductividad térmica $0.12-0.25$ $\mathrm{W} /(\mathrm{K} \cdot \mathrm{m})[6-8]$.

3. Manguera CPVC. Temperatura máxima de operación $82.2^{\circ} \mathrm{C}$, presión máxima: $1 \mathrm{Mpa}$ (100 Psi); Mínima resistencia hidrostática a la rotura: $2 \mathrm{Mpa}$ (200 Psi); conductividad térmica: $0.14 \mathrm{~W} /(\mathrm{K} \cdot \mathrm{m})[9-10]$.

Latas de aluminio: El punto de fusión del aluminio es de 933,47 grados Kelvin o de 661,32 grados Celsius o grados centígrados. El punto de ebullición del aluminio es de 2792 grados Kelvin o de $2519,85^{\circ} \mathrm{C}$. Conductividad térmica 209$230 \mathrm{~W} /(\mathrm{K} \cdot \mathrm{m})$ [11 - 13].

\subsection{Metodología para la selección del material de manguera}

3.2.1 Comprobación de resistencia térmica de las mangueras

Como parte del diseño del calentador se evaluaron tres materiales con el objetivo de comprobar su resistencia térmica. Los materiales evaluados fueron los siguientes:
1. Caucho (Manguera de riego)

2. PVC (Manguera para nivel)

3. CPVC (Manguera CPVC).

El procedimiento para evaluar los materiales consistió en hervir en agua cada tipo de manguera individualmente por 10 min, para determinar si estos soportan o no el calor a altas temperaturas $\left(100^{\circ} \mathrm{C}\right)$.

Como resultado, la manguera 2 y 3 pasaron la prueba, no se vieron afectadas por la temperatura, en cambio la manguera 1 se deformó y comenzó a desintegrarse.

\subsubsection{Selección final del material}

Entre los materiales de manguera 2 y 3 se hicieron una serie de pruebas, con seis configuraciones (ver tabla 1), en cada ensayo se midió la temperatura del agua después de $30,60,150$ y 210 minutos expuesta al sol.

Tabla 1. Mediciones de temperatura vs tiempo

\begin{tabular}{|l|l|l|l|l|}
\hline & t 1 & t 2 & t 3 & t 4 \\
\hline $\begin{array}{l}\text { Solo la manguera } \\
\text { para nivel }\end{array}$ & 42.1 & 40.1 & 40.7 & 32.3 \\
\hline $\begin{array}{l}\text { Manguera para } \\
\text { nivel recubierta por } \\
\text { lata }\end{array}$ & 42.2 & 39.9 & 42 & 33 \\
\hline $\begin{array}{l}\text { Manguera para } \\
\text { nivel recubierta por } \\
\text { lata pintada }\end{array}$ & 42.9 & 41.9 & 39.8 & 32.2 \\
\hline $\begin{array}{l}\text { Solo manguera de } \\
\text { CPVC }\end{array}$ & 37.4 & 37.6 & 38.8 & 33.2 \\
\hline $\begin{array}{c}\text { Manguera de CPVC } \\
\text { recubierta de lata }\end{array}$ & 36.8 & 37.5 & 38.9 & 32.2 \\
\hline $\begin{array}{c}\text { Manguera CPVC } \\
\text { recubierta por lata } \\
\text { pintada }\end{array}$ & 38.9 & 41.0 & 35.5 & 33.4 \\
\hline Tiempo (min) & 30 & 60 & 150 & 210 \\
\hline
\end{tabular}

De acuerdo con los resultados mostrados en la tabla 1, tomando en cuenta la temperatura que se tomó del calentamiento del agua dentro del interior de las mangueras con cada una de sus especificaciones, la más prometedora fue la manguera de CPVC, ya que después de pasar las horas expuestas al sol, la temperatura del agua se mantuvo. Sin embargo, en la manguera PVC la temperatura del agua aumentó rápidamente en un periodo de tiempo corto de $30 \mathrm{~min}$. Al ser más económica y al tenerla disponible elegimos la manguera de PVC porque cumple con los requerimientos.

\section{Diseño de prototipo del calentador}

Las características generales que debe reunir un captador solar térmico son las siguientes:

- Resistente a las condiciones exteriores (polvo, humedad, etc.)

- Resistente a temperaturas altas y bajas

- Estable y duradero 
- Fácil de montar

- Eficiente en la conversión de energía [1].

El prototipo del calentador consta de cuatro componentes:

1. El Marco de aluminio

2. La base de zinc

3. El circuito de serpentín

4. El vidrio.

En la figura 1 se muestra los componentes (realizado en Google SketchUP 8).

El calentador consiste en una caja, pintada de negro en el exterior. Con el objetivo de absorber el calor y mantenerlo dentro por la refracción del aluminio.

El calentador cuenta con dos entradas y una salida. El agua pasa a través de la manguera que está recubierta por las latas de soda pintadas de negro. La flexibilidad de la manguera nos permitió doblar el circuito y con la forma del zinc (corre agua), el cual ayuda en alinear las latas sobre este.

El circuito tiene formas de serpentines en "S", son dos serpentines, uno paralelo al otro. Se escogió esta configuración para el ahorro de materiales como lo son las uniones y facilitar el recorrido del agua hacia la salida.

El circuito se introduce dentro de la caja y se tapa con un vidrio, para evitar cualquier interferencia en la transferencia de calor por los efectos de la convección.

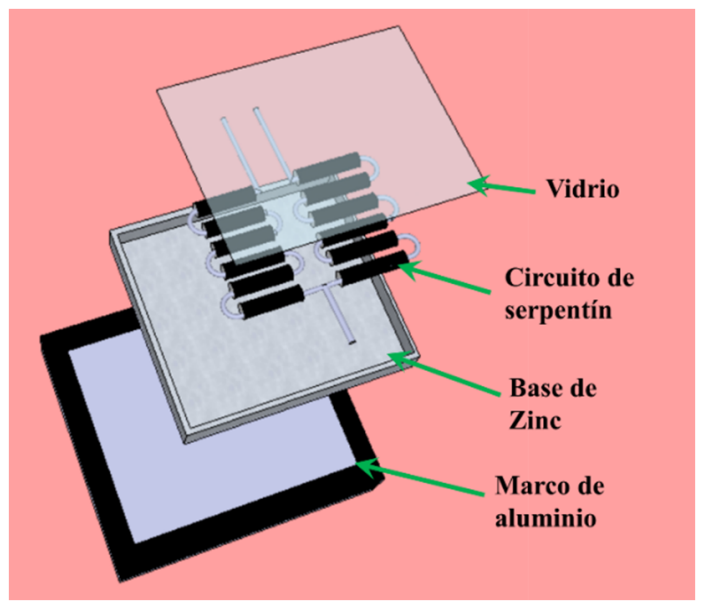

Figura 1. Diseño de prototipo del calentador solar.

\section{Funcionamiento del calentador}

El funcionamiento de nuestro prototipo aprovecha la curva de transitividad espectral del vidrio, ya que con los espesores de vidrio que se encuentran en la práctica transmiten más del $90 \%$ de la radiación en el rango visible y es prácticamente opaco (no transparente) a la radiación en las regiones infrarrojas de longitudes de onda más largas del espectro electromagnético. Como consecuencia, el vidrio permite que, entre la radiación solar, pero no deja que escape la radiación infrarroja proveniente de las superficies interiores. Esto causa la elevación en la temperatura en el interior como resultado de la energía que se acumula. Por este motivo se utilizó el vidrio en la parte frontal para aprovechar este efecto (efecto invernadero).

Luego que esta radiación es transmitida por el vidrio es absorbida por las latas de aluminio (de buena conductiva térmica) pintadas de negro que absorben casi toda la radiación térmica. Esta energía térmica es transmitida a la manguera por donde circula el agua por medio de la conducción y de la manguera al agua por convección [14].

\section{Orientación del calentador}

El calentador fue orientado hacia el sur, con una inclinación de $10^{\circ}$ con respecto a la horizontal. Para la orientación se consultaron los datos de los paneles solares del Parque Solar en Sarigua. Esta posición supone el máximo aprovechamiento de la radiación solar (alrededor de 10 horas diarias de la radiación solar). El comportamiento de la radiación es de orden ascendente de 6:00 a. m. - 12:30 p. m. luego es descendente hasta un horario de 06:30 p. m. [15 - 16].

Es importante indicar que, para su mayor aprovechamiento solar, el calentador no debe estar debajo de objetos que le den sombra.

\section{Resultados}

Tabla 2. Resultados de mediciones del prototipo

\begin{tabular}{|c|c|c|c|}
\hline & Hora & $\begin{array}{c}\text { T }\left({ }^{\circ} \mathbf{C}\right) \\
\text { ambiente }\end{array}$ & $\begin{array}{c}\text { T }\left({ }^{\circ} \mathbf{C}\right) \text { salida } \\
\text { de agua del } \\
\text { circuito }\end{array}$ \\
\hline \multicolumn{4}{|c|}{ Domingo 10 de junio } \\
\hline 1 & $1: 41$ p. m. & 36.6 & 43.6 \\
\hline 2 & $2: 40$ p. m. & 32.5 & 45.8 \\
\hline 3 & $3: 40$ p. m. & 32.5 & 47.7 \\
\hline 4 & $5: 00$ p. m. & 31.1 & 39.0 \\
\hline \multicolumn{5}{|c|}{ Sábado 23 de junio } \\
\hline 1 & $11: 00$ a. m. & 36.3 & 38.8 \\
\hline 2 & $12: 00$ m. d. & 36.6 & 41.3 \\
\hline
\end{tabular}

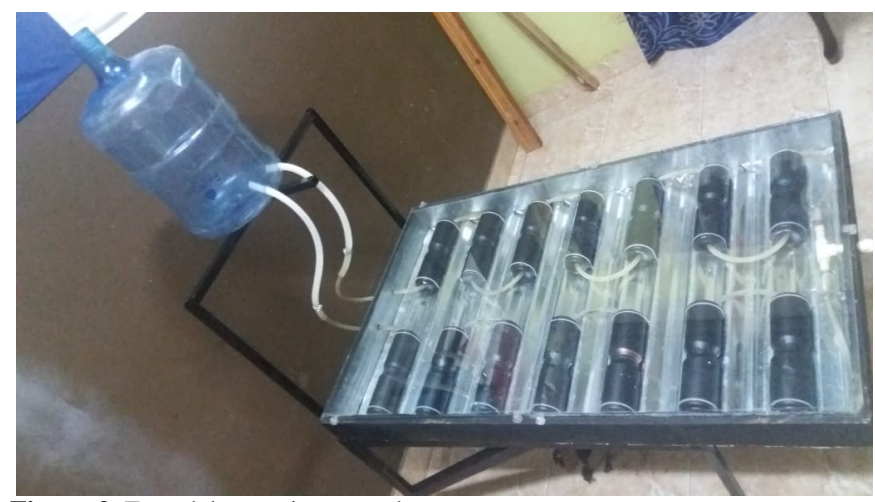

Figura 2. Foto del prototipo armado. 


\section{Conclusiones}

Para la selección final del material de la manguera, nos concentramos en el material que más rápido calentó el agua y no por el que mantuvo por más tiempo la temperatura del agua.

En la parte de resultado en la medición de las 2:40 p. m. estaba nublado y aún así el agua salió caliente, haciendo que este calentador sea estable con la variación en el clima.

Como trabajo a futuro queda analizar la eficiencia del calentador, esto implica un análisis de las condiciones climáticas, incidencia del sol en el calentador, balance de energía y transferencia de calor.

\section{AGRADECIMIENTO}

Al profesor Roberto Cigarruista por los consejos sobre la elección del material para el calentador, al Lic. Jorge Frago por aportación de unas ideas al prototipo, y a la institución y colaboradores de la Universidad Tecnológica de Panamá por permitirnos usar el área de trabajos, a nuestra familia por ayudarnos en la recolección de los materiales y también sus apoyos en la construcción del prototipo.

\section{REFERENCIAS}

[1] J. A. Carta González, R. Calero Pérez, A. Colmenar Santos, M. A. Castro Gil. Centrales de Energía Renovable: generación eléctrica con energías renovables. Madrid: Pearson Educación, S.A., 2009.

[2] Calentadores Solares | Unidad de Ecotecnologías, UNAM, Campus Morelia. (2018). Ecotec.unam.mx. [Online] Available: http://ecotec.unam.mx/Ecotec/ecoteca/calentadores-solares-2.

[3] TOTCLINIC. (2012, May 14). ¿Qué es un termómetro digital? y ¿Cómo se usa? [Online] Available: http://www.totclinic.com/blog/termometros/que-estermometro-digital-como-se-usa/.

[4] (2011, Nov 10). Los termómetros: Funcionamiento y tipos | QuimiNet.com. [Online] Available: https://www.quiminet.com/articulos/los-termometrosfuncionamiento-y-tipos-2637622.htm.

[5] Rainbird (2016). [Online] Available: http://www.rainbird.com/sites/default/files/media/documents/2 018-01/ts_A5PC_sp.pdf.

[6] Manguera de nivel 3/8" rollo 100m. (2018). [Online] Available: http://tienda.urrea.com/Catalogo/es/urrea/122277-Manguerade-nivel-38-rollo-100m.

[7] Jq.com. ar. (2018). PVC - Datos Tecnicos [online] Available: http://www.jq.com.ar/imagenes/productos/pvc/pvcprop/dtecnic os.

[8] Lehengoak S.A. [online] Available: http://www.lehengoak.com/pdfscatalogo/Mangueras\%20Industriales\%20transparentes.pdf.

[9] Sites.google.com. (2018). TUBO CPVC 1/2" - Especificaciones Técnicas para Construcción de Viviendas. [online] Available: https://sites.google.com/a/correo.udistrital.edu.co/manualvivie ndas/3-especificaciones-de-materiales/tuberia-hidraulica-ysanitaria/tubo-cpvc-1-2.

[10] PVC clorado (CPVC). (2013, nov 23). Available: http://tecnologiadelosplasticos.blogspot.com/2013/11/pvcclorado-cpvc.html.
[11] Aluminio - Propiedades del aluminio. (2009). Available: https://elementos.org.es/aluminio.

[12] Propiedades del Aluminio — Universidad de Cádiz Tablaperiodica.uca.es. [online] Available: http://tablaperiodica.uca.es/Tabla/elementos/Aluminio/Grupo1 /Prop.\%20Al.

[13] L. Laiz Trujillo. (2010, may 14). Latas de aluminio. Avaible: http://materialesdeingenieriaindustrial.blogspot.com/2010/05/1 atas-de-aluminio.html.

[14] Yunus A. Cengel, Afshin J. Ghajar. Transferencia de Calor y Masa. 4ta edición. Universidad de Nevada.McGraw Hill.

[15] A. García. (2014, nov 29). Gira Técnica a la Planta Solar en el Desierto de Sarigua. Available: from http://panamahitek.com/gira-tecnica-la-planta-solar-en-eldesierto-de-sarigua/.

[16] Empresa de Generación Eléctrica S. A. PLANTA SOLAR SARIGUA. Available: http://www.irena.org/eventdocs/RRA_and_CECCA_Panama/ Antonio\%20Clement-EGESA.pdf. 